\title{
Flúter IC
}

\author{
Pablo Franquelo Morales ${ }^{a}$, César Canales Hortelano \\ Javier Viñas González $z^{\mathrm{b}}$, Beatriz Valero Serrano ${ }^{\mathrm{a}}$
}

\begin{abstract}
a Servicio de Urgencias, Hospital Virgen de la Luz, Cuenca, España.

b Servicio de Cardiología, Hospital Virgen de la Luz, Cuenca, España.

Correspondencia: Pablo Franquelo Morales, Servicio de Urgencias, Hospital Virgen de la Luz, C/ Hermandad de Donantes de Sangre $n^{\circ} 2,16002-C u e n c a$, España. Correo electrónico: pfranquelo@yahoo.es.
\end{abstract}

Recibido el 20 de septiembre del 2011.

Aceptado para su publicación el 15 de octubre de 2011.

\section{RESUMEN}

La presencia de fármacos antiarrítmicos en los tratamientos habituales de los pacientes es un hecho frecuente en las consultas de Atención Primaria, fundamentalmente en aquellos con diagnósticos de fibrilación auricular y flúter, que generalmente precisan medicación de forma indefinida. Los efectos secundarios derivados de su utilización son múltiples y variados y, aunque se encuentran ampliamente descritos en la literatura, conviene conocerlos por su uso habitual y su potencial peligro. La flecainida es un antiarrítmico de clase IC, de uso común en pacientes con fibrilación auricular, que puede presentar un efecto proarrítmico como complicación. Presentamos el caso de un paciente con diagnóstico de fibrilación auricular en tratamiento con flecainida que derivó en una taquicardia regular con QRS ancho por conducción aurículo-ventricular 1:1, conocido como flúter IC, provocando inicialmente confusión en el diagnóstico.

Palabras clave. Flecainida, Fibrilación Auricular, Agentes Antiarrítmicos, Flúter Auricular.

\section{ABSTRACT}

\section{Class IC Atrial Flutter}

Antiarrythmic drugs commonly form part of the treatment regimens of primary care patients, especially in those with diagnosis of atrial fibrillation and flutter, conditions which usually require medication indefinitely. The side effects from their use are multiple and varied, and although these are widely described in the literature, it is advisable to be familiar with adverse effects, due to the frequent use of antiarrythmics and their potential danger. For example, flecainide, a class IC antiarrhythmic agent commonly used in patients with atrial fibrillation, can have proarrhythmic effects. We report the case of a patient previously diagnosed with atrial fibrillation and treated with flecainide, who developed regular wide QRS tachycardia with 1:1 atrioventricular conduction, known as class IC atrial flutter, causing initial confusion in diagnosis.

Key words. Flecainide, Atrial Fibrillation, Anti-arrhythmia Agents, Atrial Flutter.

\section{INTRODUCCIÓN}

Hoy día es frecuente la presencia de fármacos antiarrítmicos en los tratamientos habituales de los pacientes de Atención Primaria, fundamentalmente en aquellos con diagnósticos de fibrilación auricular (FA) y flúter, que generalmente precisan medicación por largo tiempo o de una forma indefinida. No obstante, desde finales de los 80 , con el estudio CAST ${ }^{1}$ las perspectivas del tratamiento antiarrítmico cambiaron sustancialmente tras conocerse los efectos secundarios derivados de su utilización. La flecainida $(\mathrm{Fc})$ es un antiarrítmico de clase IC, de uso común en pacientes con FA, capaz de presentar un efecto proarrítmico como complicación que puede tener consecuencias fatales ${ }^{2}$. Presentamos el caso de un paciente con FA permanente en tratamiento con Fc que derivó en lo que se conoce como flúter $\mathrm{IC}^{3}$. Esta entidad, con una incidencia muy baja, es provocada por el efecto dependiente de uso de la Fc, es decir, su efecto se potencia a una frecuencia cardiaca alta, aumentando secundariamente la frecuencia ventricular, lo que provoca una conducción aurículo-ventricular 1:1, que es todavía más excepcional ${ }^{4}$, y puede generar confusión en su diagnóstico. Profundizamos en el concepto de proarritmia farmacológica, como una complicación del uso de algunos antiarrítmicos, a través de la aparición de un tipo de flúter atípico. 


\section{OBSERVACIONES CLÍNICAS}

Varón de 68 años que consultó por palpitaciones de reciente comienzo, sin mareo ni afectación del estado general. Se practicó un electrocardiograma (EKG) con presencia de una taquicardia regular de QRS ancho a unos 200 lpm, interpretada inicialmente como de origen ventricular. No obstante, comprobada la buena tolerabilidad del paciente, que contrastaba con aquel patrón electrocardiográfico, replanteamos el diagnóstico inicial, contemplando una conducción aberrante en una taquicardia de
QRS estrecho como opción más plausible. Por tanto, se optó por realizar un masaje carotideo inicialmente, que, transcurridos unos minutos, puso de manifiesto un flúter con conducción variable (figuras 1 y 2). Se optó por añadir un fármaco beta bloqueante para el control definitivo de la respuesta ventricular con buen resultado.

La exploración física mostraba una TAde $110 / 80 \mathrm{~mm} /$ $\mathrm{Hg}$ y FC de $60 \mathrm{lpm}$, con auscultación cardiopulmonar normal. El paciente refería episodios ocasionales de mareo no sincopal por los cuales se encontraba

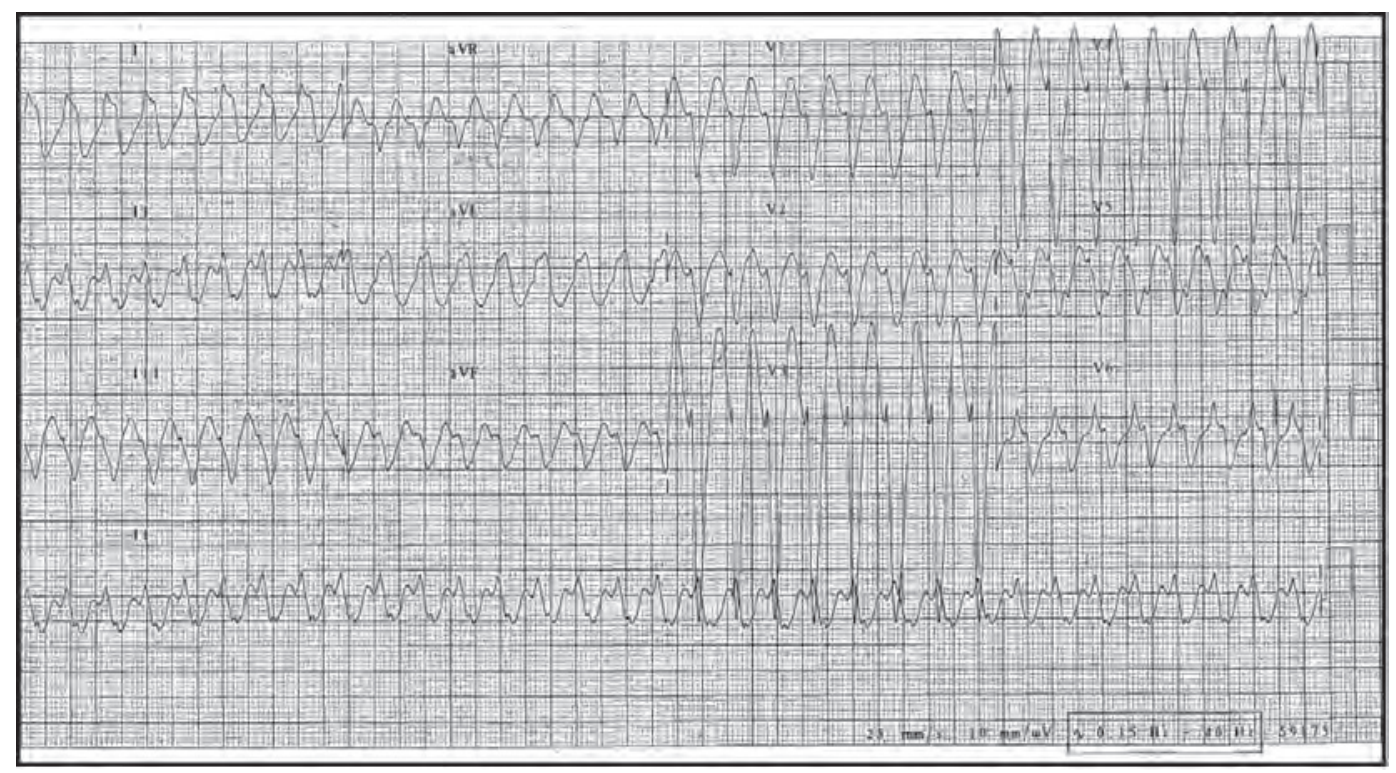

Figura 1. EKG al ingreso que muestra una taquicardia con QRS de morfología BRIHH en precordiales y eje inferior en miembros.

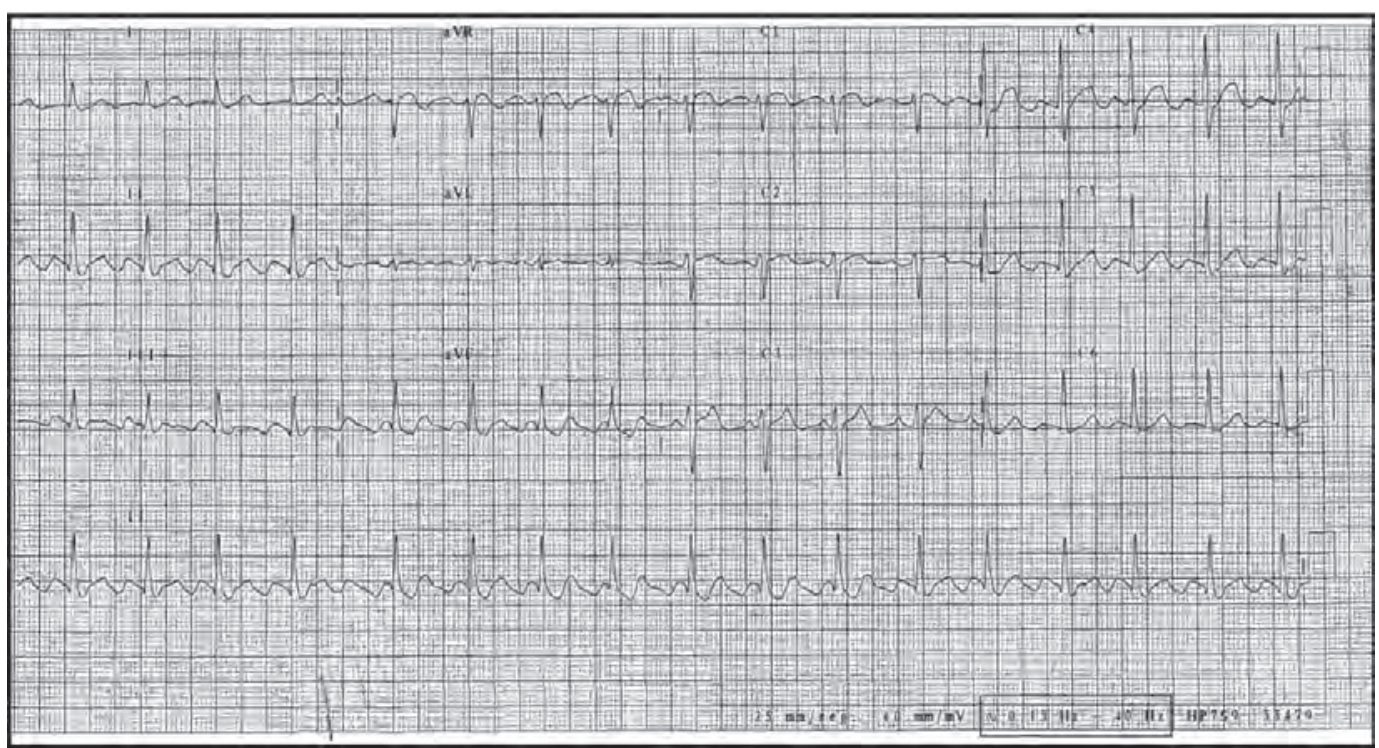

Figura 2. EKG tras masaje carotídeo con flúter auricular de morfología aparentemente común y conducción variable a los ventrículos con QRS normal. 


\begin{tabular}{|c|c|c|c|}
\hline Clase & Mecanismo de acción & Características & Principio activo \\
\hline IA & $\begin{array}{l}\text { Bloqueante de los canales } \\
\text { del } \mathrm{Na}^{+} \text {por acción / } \\
\text { disociación intermedia }\end{array}$ & $\begin{array}{l}\text { Prolongan la repolarización. } \\
\text { Anticolinérgicos. Cinética intermedia. } \\
\text { Prolongan la duración del potencial de } \\
\text { acción }\end{array}$ & $\begin{array}{l}\text { Procainamida, Ajmalina, Disopiramida, } \\
\text { Quinidina }\end{array}$ \\
\hline IB & $\begin{array}{l}\text { Bloqueante de los canales } \\
\text { del } \mathrm{Na}^{+} \text {por acción/ } \\
\text { disociación rápida }\end{array}$ & $\begin{array}{l}\text { Cinética rápida. Reducen o acortan el } \\
\text { potencial de acción }\end{array}$ & $\begin{array}{l}\text { Lidocaina, Mexiletina, Tocainida, } \\
\text { Fenitoina }\end{array}$ \\
\hline IC & $\begin{array}{l}\text { Bloqueante de los canales } \\
\text { del } \mathrm{Na}^{+} \text {por acción/ } \\
\text { disociación lenta }\end{array}$ & $\begin{array}{l}\text { Reducen la velocidad de conducción. } \\
\text { Cinética lenta. Prolongan ligeramente el } \\
\text { potencial de acción }\end{array}$ & $\begin{array}{l}\text { Propafenona, Flecainida, Encainida, } \\
\text { Morizicina }\end{array}$ \\
\hline II & $\begin{array}{l}\text { Bloqueantes de receptores } \\
\text { adrenérgicos beta }\end{array}$ & Disminuyen el automatismo & $\begin{array}{l}\text { Propranolol, Metoprolol, Nadolol, } \\
\text { Timolol, Sotalol, Atenolol, Esmolol }\end{array}$ \\
\hline III & $\begin{array}{l}\text { Bloqueante de los canales } \\
\text { del } \mathrm{K}^{+}\end{array}$ & $\begin{array}{l}\text { Prolongan la repolarización y } \\
\text { duración del potencial de acción. } \\
\text { Antiadrenérgicos }\end{array}$ & $\begin{array}{l}\text { Bretilio, Amiodarona, Sotalol, } \\
\text { Azimilida, Dronedarona, Ibutilida, } \\
\text { Dofetilida, Nibentan, Clofilium, } \\
\text { Tedisamil, Sematilida, NAPA(N- } \\
\text { acetilprocainamida) }\end{array}$ \\
\hline IV & $\begin{array}{l}\text { Bloqueantes de los canales } \\
\text { del } \mathrm{Ca}^{2+}\end{array}$ & $\begin{array}{l}\text { Deprimen la función de células } \\
\text { Ca-dependientes (nodos sinusal y } \\
\text { auriculoventicular) }\end{array}$ & $\begin{array}{l}\text { Verapamilo, Diltiazem, Bepridil, } \\
\text { Mibefradil }\end{array}$ \\
\hline Digitálicos & Aumento del tono vagal & $\begin{array}{l}\text { Disminuye el automatismo y la } \\
\text { velocidad de conducción }\end{array}$ & Digoxina, Digitoxina, B-metil-digoxina \\
\hline Otros & $\begin{array}{l}\text { Agonistas receptores } \\
\text { A1(cardiacos) y } \\
\text { A2(vasculares) }\end{array}$ & Cronotrópico y dromotrópico negativos & Adenosina ATP \\
\hline
\end{tabular}

Tabla 1. Clasificación de los fármacos antiarrítmicos. Modificada de Vaughan Williams.

en estudio, con diagnóstico de FA persistente, en tratamiento reciente con Fc y acenocumarol. Las exploraciones complementarias mostraron una analítica normal, incluyendo hormonas tiroideas, sin elevación de enzimas cardiacas, y radiografía de tórax sin datos de interés. Se realizó un ecocardiograma en ritmo sinusal con ventrículos de tamaño, grosor y función sistólica normales, mínima dilatación de la aurícula derecha con insuficiencia tricuspídea ligera y gradiente ventrículo-auricular derecho de $24 \mathrm{mmHg}$.

En la evolución se mantuvo la respuesta ventricular controlada tras tratamiento frenador con atenolol, aunque a expensas de episodios de hipotensión levemente sintomática. No se documentó ningún episodio, ni siquiera no sostenido, de taquiarritmias auriculares durante el resto de su estancia hospitalaria. Ante los episodios previos de fibrilación y flúter durante el tratamiento con fármacos antiarrítmicos de tipo IC, se diagnosticó un flúter auricular 1:1 conducido con aberrancia en presencia de tratamiento con $\mathrm{Fc}$, lo que se denomina flúter IC, por lo que se suspendió definitivamente dicho fármaco. Se instauró acenocumarol de nuevo con INR entre $2-3$ y bisoprolol $5 \mathrm{mg} / 24 \mathrm{~h}$ de manera transitoria a la espera de un tratamiento más definitivo de su arritmia en función de próximos acontecimientos.

En posteriores consultas, el paciente presentó aceptable control de la frecuencia ventricular sin recurrencias, por lo que actualmente se mantiene el mismo tratamiento.

\section{COMENTARIOS}

La Fc es un fármaco antiarrítmico del grupo IC (tabla 1$)^{5}$, utilizado para el tratamiento de arritmias 
supraventriculares sin cardiopatía estructural|2,6-8. Su uso principal es la cardioversión y prevención de recurrencias en pacientes con FA, así como las taquicardias reentrantes supraventriculares, intranodal o por vía accesoria ortodrómica (tabla $2)^{9}$. Su utilización se desaconseja en pacientes con cardiopatía estructural, hipertrofia ventricular significativa, bloqueos de rama y trastornos de conducción por la posibilidad de generar una arritmia ventricular ${ }^{2}$. La dosificación de la Fc se guía por la eficacia, la tolerancia y los cambios electrocardiográficos, administrándose en dosis habitual de $100-400 \mathrm{mg} / 12$ horas. Produce por lo general pocos efectos secundarios, entre los cuales destacan visión borrosa, cefalea, náuseas, parestesias, temblor, fatiga y nerviosismo ${ }^{6}$. No

\begin{tabular}{|c|c|c|}
\hline Clase & Indicación & Efectos adversos \\
\hline $\mathrm{IA}$ & $\begin{array}{l}\text { Arritmia ventricular. Prevención de fibrilación auricular } \\
\text { paroxística recurrente iniciada por sobreactividad vagal } \\
\text { Procainamida: en el síndrome de Wolff-Parkinson-White }\end{array}$ & $\begin{array}{l}\text { Procainamida: disminuye la TA .Aumenta QRS. } \\
\text { Proarritmia }\end{array}$ \\
\hline IB & $\begin{array}{l}\text { Tratamiento y prevención durante e inmediatamente } \\
\text { después de un infarto agudo de miocardio, aunque es } \\
\text { una práctica que ahora se usa menos por el gran riesgo } \\
\text { de asistolia. Taquicardia ventricular. Fibrilación auricular }\end{array}$ & $\begin{array}{l}\text { Lidocaína: sobre el SNC (parestesias, convulsiones, } \\
\text { alteración del nivel de conciencia, agitación, visión } \\
\text { borrosa, temblores), son las más frecuentes. } \\
\text { Hipotensión.Bradicardia }\end{array}$ \\
\hline IC & $\begin{array}{l}\text { Previene la fibrilación auricular paroxística. } \\
\text { Tratamiento de taquiarrítmias recurrentes de una } \\
\text { conducción eléctrica anormal }\end{array}$ & $\begin{array}{l}\text { Flecainida: disminuye la TA. } \\
\text { Flúter 1:1.Taquicardia ventricular } \\
\text { Propafenona: disminuye la TA. Flúter 1:1.Taquicardia } \\
\text { ventricular. Insuficiencia cardiaca. Afectación } \\
\text { gastrointestinal }\end{array}$ \\
\hline II & $\begin{array}{l}\text { Disminución de la mortalidad en infartos de miocardio. } \\
\text { Prevención de taquiarrítmias recurrentes }\end{array}$ & $\begin{array}{l}\text { Disminuye la TA. Broncoespasmo. Bradicardia. Bloqueo } \\
\text { AV e insuficiencia cardiaca }\end{array}$ \\
\hline III & $\begin{array}{l}\text { En el Síndrome de Wolff-Parkinson-White } \\
\text { Sotalol: taquicardias ventriculares y fibrilación auricular } \\
\text { Nibentan: flúter auricular y fibrilación auricular }\end{array}$ & $\begin{array}{l}\text { Amiodarona: disminuye la TA. Aumenta el QT. Hiper/ } \\
\text { hipotiroidismo. Toxicidad pulmonar. Hepatotoxicidad. } \\
\text { Fotosensibilidad. Depósitos corneales. Afectación } \\
\text { cutánea. Polineuropatía. Neuropatía óptica. Interacción } \\
\text { con acenocumarol. Bradicardia. Torsade de pointes } \\
\text { Sotalol: disminuye la tensión arterial. Bloqueo AV. } \\
\text { Insuficiencia cardiaca. Prolonga QT. Torsade de pointes }\end{array}$ \\
\hline IV & $\begin{array}{l}\text { Prevención de taquicardia supraventricular paroxística } \\
\text { recurrente. Reduce la velocidad de contracción } \\
\text { ventricular en pacientes con fibrilación auricular }\end{array}$ & $\begin{array}{l}\text { Diltiacem: disminuye la TA. } \\
\text { Bloqueo AV. Insuficiencia cardiaca } \\
\text { Verapamilo: disminuye la TA. Bloqueo AV. Insuficiencia } \\
\text { cardiaca. Interacciona con digoxina }\end{array}$ \\
\hline Digitálicos & $\begin{array}{l}\text { Supresión de TPSV (de primera elección si función } \\
\text { VI deprimida). Control de la frecuencia ventricular en } \\
\text { fibrilación, flúter y taquicardia auricular( de primera } \\
\text { elección si función VI deprimida) }\end{array}$ & $\begin{array}{l}\text { Digoxina: Bloqueo AV. Bradicardia. Ginecomastia. } \\
\text { Intoxicación digitálica,(ocular, digestiva, neurológica, } \\
\text { proarrítmica) }\end{array}$ \\
\hline
\end{tabular}

Tabla 2. Utilidad clínica y efectos adversos de los principales antiarrítmicos.

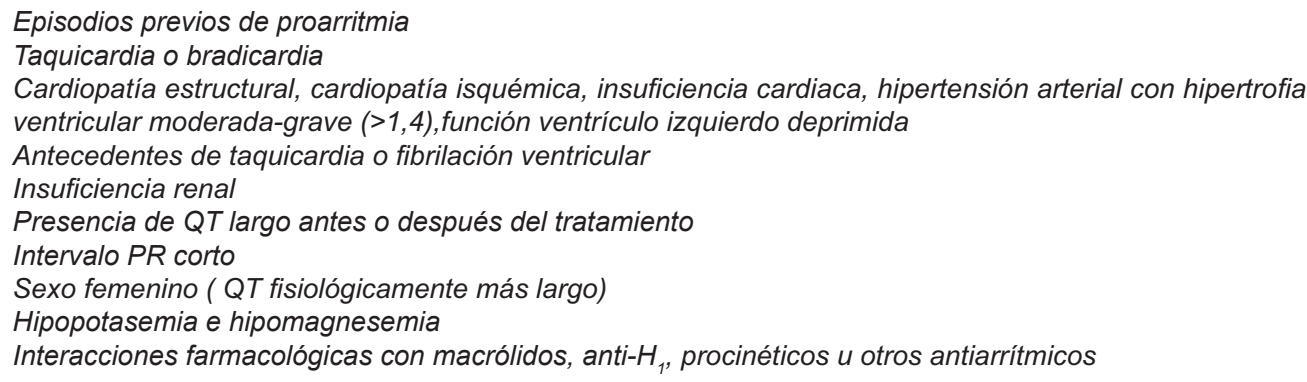

Tabla 3. Factores de riesgo para el desarrollo de proarritmia durante el tratamiento con fármacos antiarrítmicos. 
obstante, sus principales complicaciones se derivan de su efecto proarrítmico ${ }^{2,9}$, siendo más acusado en pacientes con cardiopatía estructural y en pacientes con disfunción sinusal o problemas de conducción aurículo-ventricular o post-infarto. Su manifestación en estos casos puede ser variable, desde flúter auricular con conducción 1:1 con QRS ancho, que asemeja una taquicardia ventricular, como ocurrió en nuestro paciente, hasta episodios de hipotensión arterial, insuficiencia cardiaca o incluso una taquicardia ventricular incesante, entre otros.

En ocasiones la Fc, debido a su efecto en refractariedad y velocidad de conducción, es capaz de enlentecer la frecuencia de un flúter auricular preexistente ${ }^{10,11}$, además de convertir la FA en un flúter auricular, a lo que se denomina flúter IC. Esta arritmia puede presentar una conducción 1:1 por su efecto dependiente de uso, aumentando la frecuencia ventricular con presencia de un ensanchamiento del QRS en el registro electrocardiográfico. Esta aberrancia en la conducción resulta indistinguible de una taquicardia ventricular en un electrocardiograma, por lo que es fundamental para diagnosticarla tener un alto nivel de sospecha en aquellos pacientes con FA tratados con fármacos del grupo IC y con un corazón sano ${ }^{9}$. Para prevenir esta conducción 1:1, se recomienda utilizar de forma simultánea agentes bloqueadores del nodo AV, como antagonistas del calcio, bloqueadores beta o digoxina ${ }^{2,12}$.

La proarritmia farmacológica se define como la aparición de una arritmia o el agravamiento de una preexistente como consecuencia del tratamiento con un fármaco a dosis habituales. Aunque supone un efecto secundario poco habitual de algunos fármacos antiarrítmicos, conviene tenerlo presente a la hora de utilizarlos ${ }^{9}$. Esto obliga necesariamente a evaluar los factores de riesgo que existen para su desarrollo antes de administrarlos (tabla 3$)^{9}$. Del mismo modo, evitar la politerapia antiarrítmica y evaluar las interacciones con otros fármacos con efecto proarrítmico constituyen los puntos clave antes de iniciar tratamiento.

Con este caso de proarritmia secundaria al uso de Fc, pretendemos poner en conocimiento del médico de Atención Primaria las características de los principales fármacos antiarrítmicos y sus efectos secundarios, así como destacar la importancia de seleccionarlos de forma adecuada e individualizada al tratar las arritmias.

\section{BIBLIOGRAFÍA}

1. The Cardiac Arrhythmia Supresión Trial (CAST) Investigators preliminary report: effect of encainide and flecainide on mortality in a randomized trial of arrhythmia suppression after myocardial infarction. $\mathrm{N}$ Engl $\mathrm{J}$ Med. 1989; 321:406-12.

2. Castillo Domínguez JC, Cejudo Díaz del Campo L, Anguita Sánchez M. Tratamiento farmacológico de las taquiarritmias. Clasificación y características de los fármacos antiarrítmicos. Medicine. 2009; 10:2555-62.

3. García Cosío F, Pastor A, Núñez A, Magalhaes AP, Awamleh P. Flúter auricular: perspectiva clínica actual. Rev Esp Cardiol 2006; 59:816-31.

4. Taylor R, Gandhi MM, Lloyd G. Tachycardia due to atrial flutter with rapid $1: 1$ conduction following treatment of atrial fibrillation with flecainide. BMJ. 2010; 340:b4684. doi: 10.1136/bmj.b4684.

5. Vaughan Williams EM. Classification of antidysrhythmic drugs. Pharmacol Ther B. 1975; 1:115-38.

6. Benito L, Hoyo J, Montroig A, Fornés B, Fluxà G, Martí D, et al. Estudio sobre los efectos adversos de los fármacos antiarrítmicos en pacientes con fibrilación auricular atendidos en un Centro de Atención Primaria. Med Clin (Barc). 2011; 137:241-6.

7. Almendral Garrote J, Marín Huerta E, Medina Moreno O, Peinado Peinado R, Pérez Álvarez L, Ruiz Granell R, et al. Guías de práctica clínica de la Sociedad Española de Cardiología en arritmias cardíacas. Rev Esp Cardiol. 2001; 54:307-67.

8. Coll-Vinent Puig B, Sánchez Sánchez M, Mont Girbau L. Nuevos conceptos en el tratamiento de la fibrilación auricular. Med Clin (Barc). 2001; 117:427-37.

9. Martín Martínez A, Del Arco Galán C, Arribas Ynsaurriaga F. Arritmias cardiacas. En: Moya Mir MS, Piñera Salmerón $\mathrm{P}$, Mariné Blanco M, editores. Tomo I. Tratado de Medicina de Urgencias. Madrid: Laboratorios Menarini, S.A.; 2011. p. 534-37.

10. Nabar A, Rodriguez LM, Timmermans C, van Mechelen R, Wellens HJ. Class IC antiarrhythmic drug induced atrial flutter: electrocardiographic and electrophysiological findings and their importance for long term outcome after right atrial isthmus ablation. Heart. 2001; 85:424-9.

11. Ferrero A, Chorro FJ, Cánoves J, Mainar L, Blasco E, Such L. Efectos de la flecainida sobre las velocidades de conducción longitudinal y transversal en el miocardio ventricular. Estudio experimental. Rev Esp Cardiol. 2007; 60:315-8

12. Velilla Moliner J, Salazar González JJ, Asso Abadía A, Giménez Valverde A, Marquina Lacueva MI, Placer Peralta LJ. Taquicardia de QRS ancho tras la administración de adenosina. Emergencias. 2008; 20:359-62. 